\title{
Inflammatory myofibroblastic tumor of the urinary bladder during pregnancy: A case report
}

\author{
XIAOLEI REN, HAIBO XIA and ZHIMING GAO \\ Department of Urology, The Affiliated Hospital of Chifeng College, Chifeng, \\ Inner Mongolia Autonomous Region 024000, P.R. China
}

Received May 15, 2014; Accepted January 29, 2015

DOI: $10.3892 / \mathrm{ol} .2015 .2992$

\begin{abstract}
A 31 year-old pregnant female who had not menstruated for 31 weeks presented to The Affiliated Hospital of Chifeng College (Chifeng, China) with painless gross hematuria, which had been present for 10 days. A computed tomography scan revealed a tumor of $5.8 \times 6.3 \mathrm{~cm}$ in diameter. A biopsy was not obtained prior to surgery. Following the initial workup, the patient underwent a cesarean section and partial cystectomy of bladder. Pathological examination revealed inflammatory myofibroblastic tumor (IMT). An initial cytoscope examination was performed one year after surgery, with subsequent follow-up cystoscope examinations performed every three months, and at the time of writing the patient was alive, with no evidence of tumor recurrence. IMT of the urinary bladder is a rare benign lesion and to the best of our knowledge, this is the first reported case describing IMT of the urinary bladder during pregnancy. This study describes the process of diagnosis and management of the patient.
\end{abstract}

\section{Introduction}

Inflammatory myofibroblastic tumor (IMT) of the bladder is an uncommon benign tumor, which was initially reported by Roth in 1980 (1). IMT is a rare neoplasm of mesenchymal origin, which presents as a benign mass that is composed of fibrous tissues and myofibroblasts, with marked infiltration of inflammatory cells, particularly plasma cells. IMT most commonly occurs in the lungs of children and adolescents, however, it may occur in any part of the body (2). To date, only 30 cases of IMT have been reported in China, and only 150 cases have been reported worldwide (3). The occurrence of bladder cancer during pregnancy is rare, with an incidence rate of $0.235 \%(4,5)$ and IMT of the urinary bladder during

Correspondence to: Mr. Xiaolei Ren, Department of Urology, The Affiliated Hospital of Chifeng College, 42 Tianyi Road, Chifeng, Inner Mongolia Autonomous Region 024000, P.R. China

E-mail: rxlpla@163.com

Key words: bladder tumor, inflammatory myofibroblastic tumor, pregnancy pregnancy is even more rare. Radical resection is the treatment of choice for patients with IMT in this region, to prevent local recurrence (6). The choice between transurethral resection and partial cystectomy depends on the depth of the tumor invasion, however complete surgical resection is important in order to avoid local recurrence (6-8). Patients with IMT of the urinary bladder may undergo computed tomography (CT) scanning and a cystoscopy in order to obtain a biopsy prior to surgery to aid with diagnosis $(7,9,10)$. Local tumor recurrence for IMT of the urinary bladder is $4 \%$, with a good patient prognosis following complete surgical resectioning (11). To the best of our knowledge, no study concerning IMT of the urinary bladder during pregnancy has been previously reported. The current study describes the case of a 31-year-old pregnant female with IMT of the urinary bladder. Written informed consent was obtained from the patient.

\section{Case report}

A 31 year-old pregnant female who had not menstruated for 31 weeks, presented to The Affiliated Hospital of Chifeng College (Chifeng, China) on December 7th, 2013 with painless gross hematuria which had been present for 10 days. The patient did not have dysuria, a fever or weight loss and there was no previous or family history of urologic neoplasms. Physical examination indicated that the patient was anemic (hemoglobin plasma level, $37 \mathrm{~g} / \mathrm{l}$; normal range, 110-160 g/l) and the blood pressure was low $(88 / 62 \mathrm{mmHg}$; normal range $90-139 / 60-89 \mathrm{mmHg}$ ). The fetal heart rate (FHR) was 160 beats per minute (normal range, $110-160$ beats per minute). The routine blood examination indicated that the hemoglobin was $37 \mathrm{~g} / 1$. A tumor of $8.0 \times 5.3 \mathrm{~cm}$ in diameter was identified in the right and front wall of bladder on ultrasound. The ultrasound also indicated normal fetal development. Pelvic CT imaging revealed a $5.8 \times 6.3 \mathrm{~cm}$ tumor in the bladder, with an enhanced-CT value of 40.4-60.5 HU (Fig. 1). The patient received a blood transfusion and also received a continuous bladder irrigation to avoid obstruction of the catheter, which prevented blood clot formation. Cystoscopy was not performed due to the patient's weak physical condition and the associated risk of a premature birth and subsequently, a biopsy was not obtained prior to surgery. Following the initial workup, on December 13th, 2013 a cesarean section was performed as well as the partial cystectomy of bladder. A male infant of 


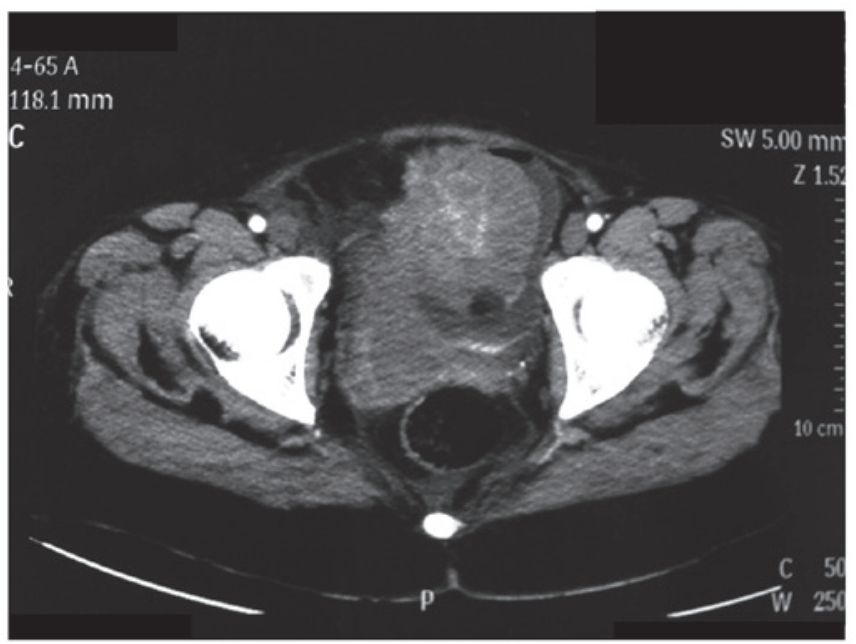

Figure 1. CT imaging revealed a tumor in the bladder, with an enhanced CT value of 40.4-60.5 HU. CT, computed tomography.

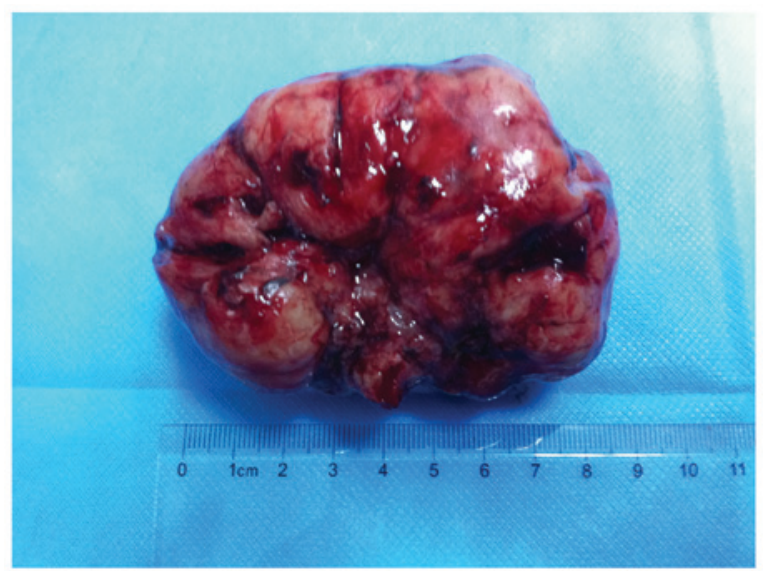

Figure 2. The tumor which was removed from the patient's bladder was $8.0 \times 7.0 \mathrm{~cm}$ in diameter.

$1.5 \mathrm{~kg}$ was born and a $8.0 \times 7.0 \mathrm{~cm}$ tumor was removed from the bladder (Fig. 2). The pathological report indicated that the growth was IMT of the urinary bladder (Fig. 3) and a positive cellular reaction for CD (34), CD (68), P (53), SMA and $\alpha$-AT was demonstrated by immunohistochemistry. The patient was discharged from hospital one month following the surgery. A follow-up cystoscopy examination was performed one year after surgery, with subsequent follow-up cystoscope examinations performed every three months, which revealed no tumor recurrence.

\section{Discussion}

IMT is a rare neoplasm, which was initially reported by Bahadori and Liebow (12) in a lung lesion in 1973. IMT can affect any age group, however, it is more common in infants and young adults, with $93 \%$ of IMT cases occuring in individuals aged $<20$ years (13). Cases of IMT have been described in almost all regions of the body and the most common site for IMT is the lung (14). If present in the genitourinary tract, this tumor type most commonly occurs is the urinary bladder (15). The exact etiology of IMT is unknown,

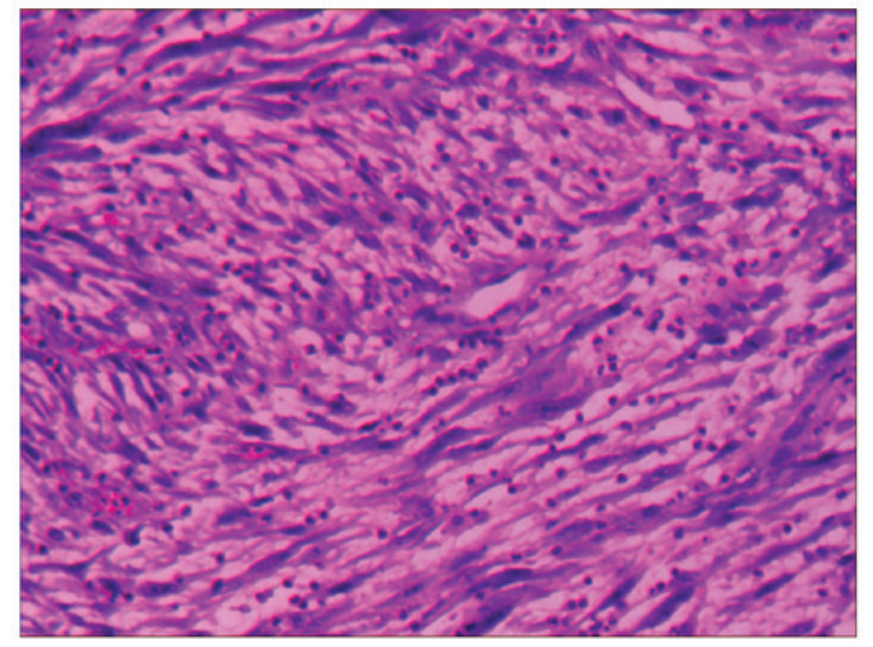

Figure 3. Hematoxylin and eosin staining confirmed that the tumor was an inflammatory myofibroblastic tumor.

however, it is hypothesized to occur secondary to infection, trauma or surgery $(16,17)$. IMT is often difficult to differentiate from malignant tumors including teratoma, liposarcoma and lymphoma prior to surgery, due to the nonspecific radiological features exhibited by the tumor (18). Urological tumors are extremely uncommon in pregnancy and no other instances of this condition have been previously reported. Preoperative examination is of great importance when IMT during pregnancy is suspected; this must include ultrasound, cystoscopy and CT. Cystoscopy is particularly important as it may indicate the size and position of the tumor. Additionally, pathological results may also be obtained by cystoscopy. Furthermore, cystoscopy does not cause harm to the patient or fetus (4). The most common method of treatment for IMT of bladder is partial cystectomy of the bladder or transurethral resection of the tumor, which prevents local recurrence (19). Intravesical therapy is controversial following surgery. IMT exhibits aggressive behavior, therefore it may be confused with malignancy. The method of determining a definitive diagnosis for IMT is pathological examination; additionally, histologically IMT is characterized by myofibroblastic spindle cells and inflammatory cells. The recurrence rate of IMT is $25 \%$ (20), and due to this significant local recurrence rate, follow-up after surgery is required.

To the best of our knowledge, this study presents the first case of IMT of the urinary bladder during pregnancy to be reported. Thus, future studies which investigate the diagnosis and treatment of the disease are required to increase knowledge with regard to this rare disease and the prognosis of such following surgery.

\section{References}

1. Roth JA: Reactive pseudosarcomatous response in urinary bladder. Urology 16: 635-637, 1980.

2. Ozsan I, Ozsoy M, Sahin E, et al: Inflammatory myofibroblastic tumor of the gallblader. Balkan Med J 30: 323-326, 2013.

3. Bo Liu, Jihong Liu, Changzhe Ke, et al: Inflammatory myofibroblastic tumor of urinary bladder: a case report and review of the literature. Chin J Urol 5: 335-337, 2007.

4. Mitra S, Williamson JG, Bullock KN and Arends M: Bladder cancer in pregnancy. J Obstet Gynaecol 23: 440-442, 2003. 
5. Hendry WF: Management of urological tumors in pregnancy. $\mathrm{Br}$ J Urol 80 (Suppl 1): 24-28, 1997.

6. Kim HW, Choi YH, Kang SM, et al: Malignant inflammatory myofibroblastic tumor of the bladder with rapid progression. Korean J Urol 53: 657-661, 2012.

7. Wu S, Chen L, Wan Q, et al: Inflammatory myofibroblastic tumor of the urinary bladder in a patient with the left renal cell carcinoma: A case report. Exp There Med 7: 1010-1012, 2014.

8. Machioka K, Kitagawa Y, Izumi K, et al: Inflammatory myofibroblastic tumor of the urinary bladder with benign pelvic lymph node enlargement: a case report. Case Rep Oncol 7: 571-575, 2014.

9. Pradhan MR, Ranjan P, Rao RN, et al: Inflammatory myofibroblastic tumor of the urinary bladder managed by laparoscopic partial cystectomy. Korean J Urol 54: 797-800, 2013.

10. Yi XL, Lu HY, Wu YX, et al: Inflammatory myofibroblastic tumor with extensive involvement of the bladder in an adolescent: a case report. World J Surg Oncol 11: 206, 2013.

11. Teoh JY, Chan NH, Cheung HY, et al: Inflammatory myofibroblastic tumors of the urinary bladder: a systematic review. Urology 84: 503-508, 2014.

12. Bahadori $\mathrm{M}$ and Liebow AA: Plasma cell granulomas of the lung. Cancer 31: 191-208, 1973.

13. Sanders BM, West KW, Gingalewski C, et al: Inflammatory pseudotumor of the alimentary tract: clinical and surgical experience. J Pediatr Surg 36: 169-173, 2001.
14. Coffin CM, Watterson J,Priest JR and Dehner LP: Extrapulmonary inflammatory myofibroblastic tumor (inflammatory pseudotumor): A clinic pathologic and immuno-histochemical study of 84 cases. Am J Surg Pathol 19: 859-872, 1995.

15. Yagnik V, Chadha A, Chaudhari S and Patel K: Inflammatory myofibroblastic tumor of the urinary bladder. Urol Ann 2: 78-79, 2010.

16. Jones EC, Clement PB and Young RH: Inflammatory pseudotumor of the urinary bladder. A clinicopathological, immunohistochemical, ultrastructural, and flow cytometric study of 13 cases. Am J Surg Pathol 17: 264-274, 1993.

17. Hojo H, Newton WA Jr, Hamoudi AB, et al: Pseudosarcomatous myofibroblastic tumor of the urinary bladder in children: a study of 11 cases with review of the literature. An Intergroup Rhabdomyosarcoma Study. Am J Surg Pathol 19: 1224-1236, 1995.

18. Chen CK, Jan CI, Tsai JS, et al: Inflammatory myofibroblastic tumor of the lung - a case report. J Cardiothorac Surg 5: 55, 2010.

19. Inoue T, Kinoshita H, Horikoshi M, et al: A case of inflammatory myofibroblastic tumor of the urinary bladder. Hinyokika Kiyo 57: 141-145, 2011 (In Japanese).

20. Lawrence B, Perez-Atayde A, Hibbard MK, et al: TPM3-ALK and TPM4-ALK oncogenes in inflammatory myofibroblastic tumors. Am J Pathol 157: 377-384, 2000. 